\title{
Mathematical Notation, Representation, and Visualization of Musical Rhythm: A Comparative Perspective
}

\author{
Yang Liu \\ School of the Museum of Fine Arts Boston \\ Boston, MA, USA \\ E-mail: yangliu1971@gmail.com
}

\author{
Godfried T. Toussaint \\ Department of Music \\ Harvard University \\ Cambridge, MA, USA \\ E-mail: godfried@cs.mcgill.ca
}

\begin{abstract}
Several methods for the mathematical notation, representation, and visualization of musical rhythm at the symbolic level are illustrated and compared in terms of their advantages and drawbacks, as well as their suitability for particular applications.
\end{abstract}

Keywords: music, rhythm, music notation, color painting notation, music representation, acoustic-mnemonic systems, binary sequences, box notation, Schillinger notation, ancient Persian notation, necklace notation, convex polygon notation, interval-content representation, visualization.

\section{INTRODUCTION}

To the uninitiated, music and mathematics may appear as antithetical activities, one expressing the emotions of the heart in a phenomenological world, and the other exploring the precise and rigorous structures of the Platonic universe. However, their intertwining relationship has a long history that in Europe goes back to Pythagoras of Samos, who in the $6^{\text {th }}$ Century B.C. developed a musical scale based on the numerical integer ratio 3:2 [29]. The origin of using a scale consisting of twelve fundamental tones, however, appears to originate two millennia earlier in China with Huang-Ti, the Yellow Emperor, circa 2700 B. C. [38]. The advent of music notation on the other hand, whether possessing greater or lesser mathematical structure, is more recent. Wulstan [35] traces music notation back to the Babylonians in 1300 B.C., and according to West [34] it goes back to at least 1800 B.C. In recent history there has been a renewed and energetic surge in the mathematical and computational aspects of music [14], [16], [19], [23]-[33].

Music notation exists at many levels of abstraction ranging from the most concrete continuous acoustic signal (usually a waveform) through the most abstract discrete symbolic notation [7], [9], [22], to the notation of emotion by means of facial expressions [20]. Some notation systems, such as Gongche notation, popular in ancient China, mark only the pitch of the notes, and not their duration [11]. Other notation systems use characters to indicate the finger positions for specific instruments, such as the Okinawan notation system for the samisen [37], and the guitar tablature notation in the West [6]. Composers have invented a variety of notation systems for specific purposes when they felt that traditional western notation did not serve their needs. For instance, the painter and composer Michael Poast made extensive use of color in his paintings that serve as scores for his compositions, and that stimulate musical expressiveness
[8]. Nevertheless, due to space limitations we focus only on mathematical, and in particular geometric, methods for the symbolic notation of musical rhythm, and we provide a small sample of examples.

\section{MNEMONIC NOTATION}

It is well known that ordinary speech in any language possesses rhythm caused by the patterns of accents or stresses [39], [40]. Indeed, the employment of similar methods that use acoustic phonetic features of vowels and consonants appears independently in geographically distant cultures [12], [49], [50]. These systems are particularly useful for teaching rhythm, and have been an invaluable tool for transmitting rhythms in cultures based on oral traditions. For instance, the mnemonic system of syllables described in the Persian $13^{\text {th }}$ Century book kitāb al-Adwār [43] uses two syllables for the strong primary beats: $\operatorname{ta}$ and tan, for short and long beats, respectively. Similarly two additional syllables are used for secondary beats: $n a$ and nan, for short and long beats, respectively. Here the long beats last twice as long as the short beats. Using this system the clave rumba rhythm of Cuba is notated as: tanan tananan tanan tan tananan. Here the five beats of the clave rumba correspond to the five $t a$ sounds at the beginnings of the words.

\section{MATHEMATICAL NOTATION}

A variety of mathematical methods exist that are used to notate a rhythmic or melodic sequence of tones. The simplest such method for rhythms is the binary sequence, in which a " 1 " is used to denote a sound, and a " 0 " to denote a silence or rest [1]. Here both symbols represent one unit of time. Thus the clave rumba is notated as [1001000100101000]. Such a representation has obvious advantages for processing rhythms by computer, but its iconic value is minimal. An improvement is the box-notation system, widely employed by ethnomusicologists, in which the two symbols used to denote sound and silence are highly dissimilar [17], [18]. One method employs " $x$ " for sound and "-" for silence. The result is $[\mathrm{x}--\mathrm{x}---\mathrm{x}--\mathrm{x}-\mathrm{x}---]$ for the clave rumba. A second more graphical rendering of box notation actually uses boxes that are either empty (silence) or contain a symbol (sound). Different symbols may denote dissimilar sounds. For instance, Fig. 1 shows the acoustic phonetic mnemonic for the clave rumba embedded in box notation, where a black filled circle denotes a primary (strong) beat, a grey filled circle a secondary (weak) beat, and an empty box 
a silence. The actual clave rumba pattern consists of only the five strong beats.

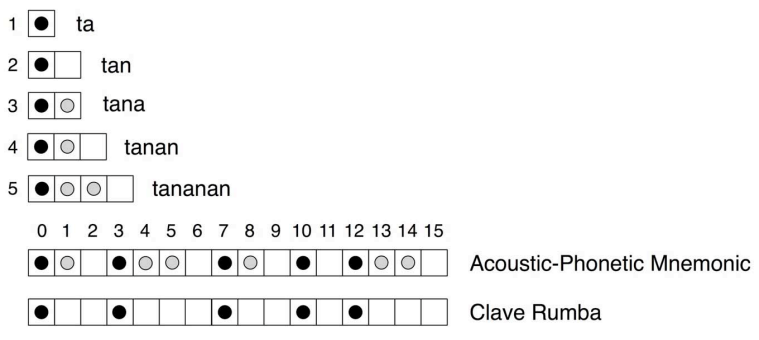

Figure 1. Acoustic phonetic mnemonic and box-notation.

The box-notation system has been generalized to handle several rhythms played simultaneously on different instruments as shown in Fig. 2. Here the white circle indicates that the hi-hat is played in the open position. This kind of notation is called drum tablature notation [1].

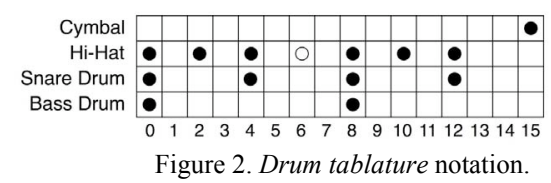

A more compact numerical notation system that favors certain algebraic approaches to analysis and composition, codifies the inter-onset intervals themselves using numbers to obtain an interval vector [2]. In this notation the clave rumba rhythm of Fig. 1 is coded as the 5-dimensional vector $[3,4,3,2,4]$. One drawback of this scheme however, is that rhythms containing different numbers of onsets yield vectors in spaces of different dimensionalities that complicate certain kinds of analyses.

\section{GEOMETRIC NOTATION}

In the early Twentieth Century in New York city, Joseph Schillinger became famous for developing a detailed and comprehensive mathematical methodology for analyzing, teaching, and composing music [4], [5], [41]. In his work he made extensive use of geometric methods. His approach to the notation of melodies in music is illustrated in Fig. 3, which shows a fragment of Haydn's Symphony No. 47 in G. In this diagram the width of each column corresponds to the duration of the shortest note employed, and the height indicates the pitch in semitones.

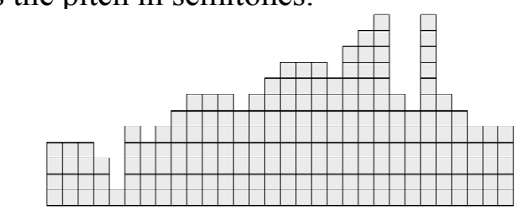

Figure 3. A Haydn piece in Schillinger music notation.

Schillinger also employed a geometric notation restricted to pure rhythm without pitch information. For this purpose he used a rectilinear curve as a function of time, the height of which alternates between two levels from the upper level to the lower level (and vice versa) at the locations of the occurrences of the note onsets. For example, the regular 4beat, 16-pulse rhythm with intervals [4444], and the clave son with intervals [33424] are described by the two curves shown in Fig. 4 (top and bottom, respectively).

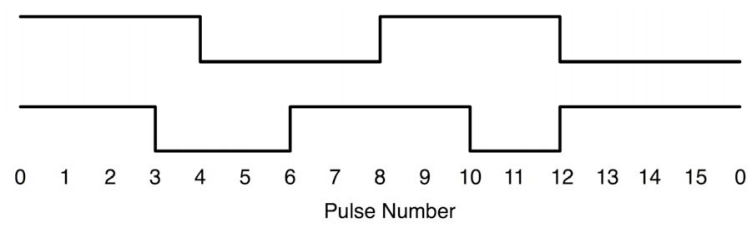

Figure 4. The clave son (bottom) in Schillinger rhythm notation.

One approach to geometrically visualizing the interval vector of a rhythm is by means of spectral notation [3]. Consider the clave rumba with interval vector [34324]. In spectral notation this vector is converted to a graph in which the vertical axis marks the durations of the inter-onset intervals, and the horizontal axis marks the onset number (index), as illustrated in Fig. 5. The upper envelope of this graph clearly highlights the pattern of variation among the inter-onset intervals as the rhythm unfolds in time.

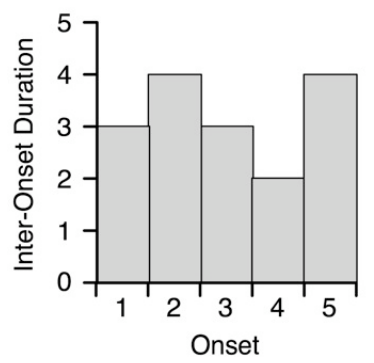

Figure 5. The clave rumba in spectral notation.

In spectral notation, at every onset one may readily observe whether the subsequent inter-onset interval is greater, smaller, or remains equal. More careful observation reveals the exact magnitude of these changes. However, from the psychological perceptual perspective, humans have more difficulty perceiving the quantitative aspects of these changes than their qualitative counterparts. For practical purposes the qualitative changes suffice. These qualitative changes are referred to as the rhythmic contour in the music theory literature [46], [47], [48]. Rhythmic contours are usually notated with the three symbols "+", "-", and "0", which for a given inter-onset interval denote, respectively, that the interval in question is greater, smaller, or has the same duration as the previous interval. Thus in contour notation the clave rumba is given by the sequence $[+--+-]$, whereas the clave son yields the contour $[0+-+-]$.

One drawback of spectral notation is that the time information along the horizontal axis is lost. To make up for this deficiency, in 1988 Gustafson introduced what he called TEDAS notation, an acronym that stands for Temporal Elements Displayed $A$ s Squares [3]. In this simple but original and effective system the durations of the inter-onset intervals are, as in spectral notation, displayed along the vertical axis, but they are also displayed along the horizontal 
axis. Therefore each inter-onset interval becomes a square, and the rhythm is displayed as a sequence of squares. For a concrete example consider the Manchu rhythm with interval vector [443122]. In TEDAS notation this rhythm becomes the graph shown in Fig. 6. This notation not only highlights the rhythmic contour of the rhythm, but it maintains its temporal accuracy, and also affords a natural way to measure rhythmic similarity [51].

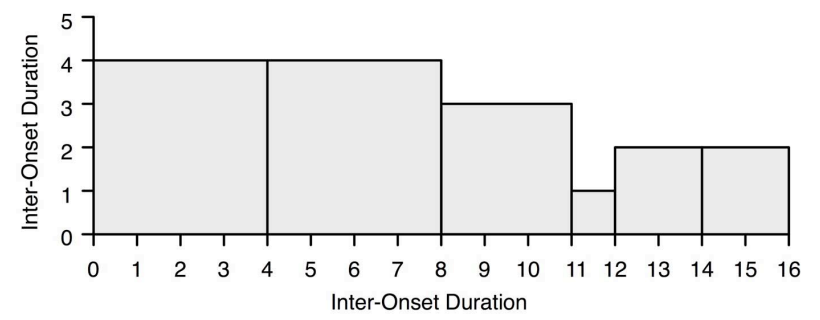

Figure 6. A 6-onset Manchu rhythm in TEDAS notation.

In 2002 Hoffman-Engl independently proposed a notation for rhythms that is almost identical to Gustsfson's TEDAS notation [10]. His chronotonic notation is illustrated in Fig. 7 with the clave rumba rhythm. Recall that the clave rumba has interval vector [34324]. In chronotonic notation the rhythm is displayed as a curve that connects a set of points with line segments. One point occurs at every pulse position in time, and its height is equal to the duration of the inter-onset interval to which it corresponds. Thus there are 3 points at height 3 , followed by 4 points at height 4 , followed by 3 points at height 3 , and so on. Hoffman-Engl defined a rhythmic similarity measure based on this notation, and reported experimental results that showed the measure was correlated with human judgments of rhythm similarity.

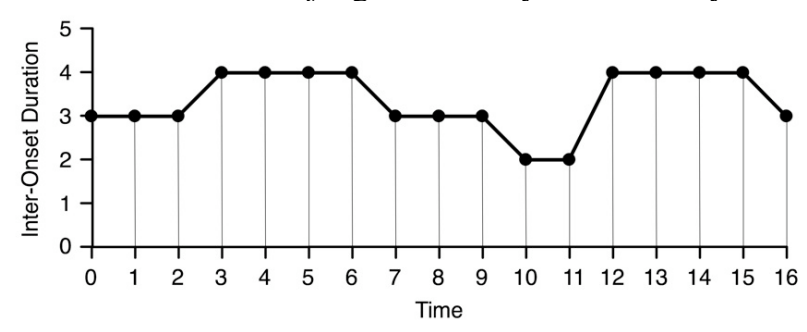

Figure 7. The clave rumba in chronotonic notation.

A natural way to notate cyclic rhythms that repeat over and over during a piece of music is by means of a circular diagram (also called a clock diagram). One of the earliest such methods was used by Safi al-Din in Thirteenth Century Bagdad in his book kitāb al-Adwār [42], [43]. An example of the clave rumba expressed in his notation is illustrated in Fig. 8. The outer circle marks off the sixteen pulses in the cycle with equally spaced small black dots. The inner circle is reserved for identifying the rhythm (in this case rumba). The sixteen pulses are separated into groups (feet) by small white circles connected with line segments to the interior circle that help to visualize the temporal structure of the rhythm. An arrow marks the start of the rhythm as well as the direction of the flow of time (counter-clockwise). Thus the inter-onset intervals marked are [34324]. The rumba clave consists of the five beats occurring at the first pulses of each of these intervals.

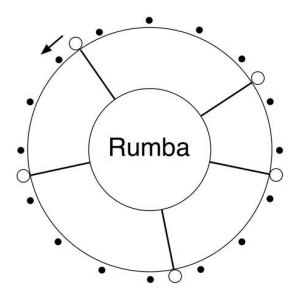

Figure 8. The clave rumba in ancient Persian notation.

Variants of circular notation have been rediscovered several times by different researchers. The typical contemporary employment of circular notation uses a "clock" diagram (also referred to as necklace notation) in which all the equally spaced pulses in the cycle are marked and numbered, the onsets of the rhythm are highlighted, and time flows in the clockwise direction [1]. In another variant (polygon notation) the onsets are connected with line segments to create a convex polygon [17], [18], [52]. Three examples of Manchu rhythms [36] in polygon notation are illustrated in Fig. 9, where black circles indicate main (strong) beats and grey circles indicate secondary (weak) beats. More recently, Benadon [45] has extended this notation by making the radial distance of a beat proportional to its duration, and has found it useful for the study of expressive timing.
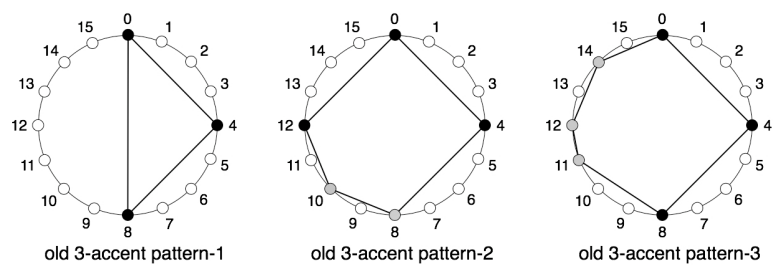

Figure 9. Examples of Manchu rhythms [36] in polygon notation.

Symmetry is an important feature of music in general and rhythm in particular [15], [21]. Polygon notation provides an effective means of visualizing the various symmetries that may be present in cyclic rhythms. Consider for example the three Manchu rhythms pictured in Fig. 9. The rhythm on the left has mirror symmetry about the line through pulses 4 and 12 , and the center rhythm about the line through pulses 2 and 10. The three strong beats in the center rhythm have mirror symmetry about the line through pulses 0 and 8 . The rhythm on the right on the other hand possesses no mirror symmetry.

\section{REPRESENTATION}

The word notation suggests that it facilitates the reading of music by a performer. However, an aspect of music may be displayed in ways that usefully illustrate some important property of music, and that may in fact impede its readability during performance. Such renderings of musical properties are perhaps better described by the word representation. In 
any case, the interdependence of notation and representation systems, and the musical information they provide has been well documented. Cohen and Katz are careful to emphasize that "no system of notation nor any kind of preservation of musical information, be it the most highly developed, is truly comprehensive" [13]. One of the most well-known and studied representations of rhythms displays a rhythm's full interval content in the form of a histogram. It is common to find music information retrieval systems that calculate global features of this histogram to characterize and classify rhythms. Consider the six-onset Manchu rhythm shown in Fig. 10 (left). The six adjacent inter-onset intervals are [443122]. In addition to these there are nine other nonadjacent inter-onset intervals indicated by line segments. The histogram of all fifteen intervals is shown on the right.

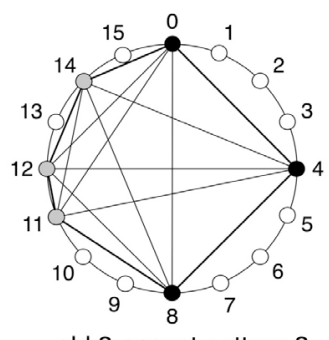

old 3-accent pattern-3

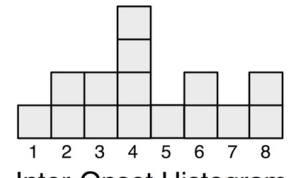

Inter-Onset Histogram
Figure 10. All the intervals of a Manchu rhythm and its histogram.

In a cyclic rhythm that lasts approximately a couple of seconds, all the pair-wise inter-onset intervals are perceived by the human mind. Therefore the interval content histogram provides instant information concerning the number and durations of these intervals. For instance, examination of the histogram in Fig. 10 reveals that apart from the four intervals of duration 4, the rest of the histogram is quite flat, indicating that the irregular aspects of this Manchu rhythm are embedded on a regular rhythmic framework consisting of beats at pulses $0,4,8$, and 12 . This representation has a severe drawback however. Consider the two 6-onset rhythms in 12-pulse cycles pictured in Fig. 11 (left and center). The two rhythms are different in the most significant way possible: one is neither a rotation nor a mirror image of a rotation of the other, i.e., geometrically the two polygons are not congruent. This is clear from the fact that in the rhythm on the left the two intervals of duration 1 are separated by two intervals of duration 2, whereas in the rhythm in the center they are separated by one interval of duration 2. Yet, surprisingly, both rhythms have exactly the same inter-onset interval histogram shown on the right. Technically these two rhythms are termed homometric. Therefore any features calculated on the histogram of these two rhythms will fail to distinguish between them. One might hope that this disconcerting example is a rare anomaly that one can safely ignore in most real-life applications. Unfortunately this is not the case. Any two non-congruent complementary rhythms with $k$ onsets and $n$ pulses such that $k=n / 2$ are homometric. This result is known as the hexachordal theorem [53]. Surprisingly, this theorem has surfaced independently in both the music theory and crystallography literatures, where several proofs of the theorem have been published [44]. One of the simplest and most elegant elementary proofs of this theorem is an induction proof due to Iglesias [54].
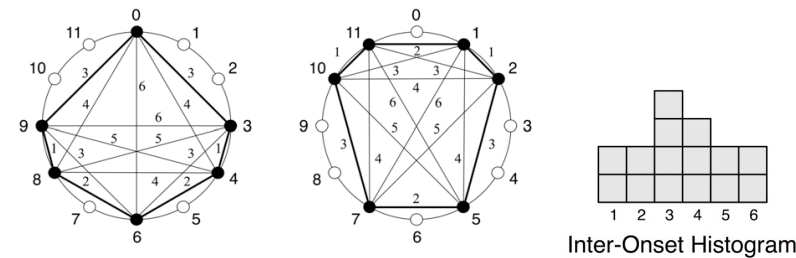

Figure 11. Two non-congruent homometric rhythms.

\section{ACKNOWLEDGMENT}

This research was financially supported by the National Sciences and Engineering Research Council of Canada (NSERC) administered through McGill University, Montreal, and by the Radcliffe Institute for Advanced Study at Harvard University, Cambridge, MA, where the second author was the Emeline Bigelow Conland Fellow during the 2009-2910 academic year.

\section{REFERENCES}

[1] W. Sethares, Rhythm and Transforms, London: SpringerVerlag, 2007.

[2] J. L. Hook, "Rhythm in the music of Messiaen: An algebraic study and an application in the Turangalila Symphony," Music Theory Spectrum, vol. 20, no. 1, April 1998, pp. 97120.

[3] K. Gustafson, "The Graphical Representation of Rhythm," Technical report, Oxford University, 1988.

[4] J. Schillinger, The Mathematical Basis of the Arts, Philosophical Library, 1948.

[5] J. Schillinger, Encyclopedia of Rhythms, Da Capo Press, 1976.

[6] M. Gaare, "Alternatives to traditional notation," Music Educators Journal, vol. 83, no. 5, March 1997, pp. 17-23.

[7] R. G. Cromleigh, "Neumes, notes, and numbers: The many methods of music notation," Music Educators Journal, vol. 64, no. 4, December 1977, pp. 30-39.

[8] M. Poast, "Visual color notation for musical expression," Leonardo, vol. 33, no. 3, 2000, pp. 215-221.

[9] R. B. Dannenberg, "Music representation issues, techniques and systems," Computer Music Journal, vol. 17, no. 3, Autumn. 1993, pp. 20-30.

[10] L. Hoffman-Engl, "Rhythmic similarity: A theoretical and empirical approach," Proc. Seventh International Conference on Music Perception and Cognition, Sydney, Australia, 2002, pp. 564-567.

[11] H. Xiangpeng and J. S. C. Lam, "Ancient tunes hidden in modern Gongchee notation," Yearbook for Traditional Music, vol. 24, 1992, pp. 8-13.

[12] D. W. Hughes, "No nonsense: The logic and power of acoustic-iconic mnemonic systems," British Journal of Ethnomusicology, vol. 9, no.2, 2000, pp. 93-120.

[13] D. Cohen and R. Katz, "The interdependence of notation systems and musical information," Yearbook of the International Folk Music Council, vol.11, 1979, pp. 100-113

[14] T. Ellingson, "The mathematics of Tibetan Rol Mo," Ethnomusicology, vol. 23, no.2, May 1979, pp. 225-243.

[15] P. Liebermann and R. Liebermann, "Symmetry in question and answer sequences in music," Computers and Mathematics with Applications, vol. 19, no. 7, 1990, pp. 59-66. 
[16] F. Sobieczky, "Results in mathematics and music: Visualization of roughness in musical consonance," Seventh IEEE Visualization, San Francisco, CA, October 27November 1, 1996.

[17] G. T. Toussaint, "The geometry of musical rhythm," In J. Akiyama, M. Kano, and X. Tan, editors, Proc. Japan Conference on Discrete and Computational Geometry, Vol. 3742, Lecture Notes in Computer Science, Springer, Berlin/Heidelberg, 2005, pp. 198-212.

[18] G. T. Toussaint, "Computational geometric aspects of musical rhythm," Abstracts 14th Fall Workshop on Computational Geometry, Massachusetts Institute of Technology, November 19-20, 2004, pp. 47-48.

[19] D. Tymoczko, A Geometry of Music: Harmony and Counterpoint in the Extended Common Practice, Oxford University Press, Oxford, UK, 2010.

[20] T. Nakanishi and T. Kitagawa, "Visualization of music impression in facial expression to represent emotion," Proc. Third Asia-Pacific Conference on Conceptual Modelling, Hobart, Australia, 2006.

[21] R. Donnini, "The visualization of music: Symmetry and asymmetry," Computers and Mathematics with Applications, vol. 12B, nos. 1/2, 1986, pp. 435-463.

[22] M. Naranjo and A. Koffi, "Geometric image modelling of the musical object," Leonardo, Supplemental Issue on Electronic Art, vol. 1, 1988, pp. 69-72.

[23] D. Wright, Mathematics and Music, American Mathematical Society, 2009.

[24] D. Temperley, Music and Probability, The MIT Press, 2007.

[25] G. Loy, Musimathics, Vol. 2: The Mathematical Foundations of Music, The MIT Press, 2007.

[26] G. Loy, Musimathics, Vol. 1: The Mathematical Foundations of Music, The MIT Press, 2006.

[27] L. Harkleroad, The Math Behind the Music, Cambridge University Press, 2006.

[28] T. H. Garland and C. V. Kahn, Math and Music: Harmonious Connections, Dale Seymour Publications, 1995.

[29] J. Fauvel, R. Flood, and R. Wilson, (editors), Music and Mathematics: From Pythagoras to Fractals, Oxford University Press, USA, 2006.

[30] D. Benson, Music: A Mathematical Offering, Cambridge University Press, 2006.

[31] E. Chew, A. Childs, and C.-H. Chuan, eds., Mathematics and Computation in Music, (Proceedings of the Second International Conference), Springer-Verlag, Berlin, 2009.

[32] T. Klouche and T. Noll, eds., Mathematics and Computation in Music, (Proceedings of the First International Conference), Springer-Verlag, Berlin, 2009.

[33] M. Keith, From Polychords to Pólya: Adventures in Musical Combinatorics, Vinculum Press, Princeton, New Jersey, 1991.

[34] M. L. West, "The Babylonian musical notation and the Hurrian melodic texts," Music \& Letters, vol. 75, no. 2., May, 1994, pp. 161-179.

[35] D. Wulstan, "The earliest musical notation," Music \& Letters, vol. 52, no. 4, October 1971, pp. 365-382.

[36] L. Li, "Mystical numbers and Manchu traditional music: A consideration of the relationship between shamanic thought and musical ideas," British Journal of Ethnomusicology, vol. 2, 1993, pp. 99-115.

[37] J. La Rue, "The Okinawan notation system," Journal of the American Musicological Society, vol. 4, no. 1, Spring 1951, pp. 27-35.

[38] K. Nakaseko, "Symbolism in ancient Chinese music theory," Journal of Music Theory, vol. 1, no. 2, November 1957, pp. 147-180.

[39] M. Liberman and A. Prince, "On stress and linguistic rhythm," Linguistic Inquiry, vol. 8, no. 2, Spring 1977, pp. 249-336.

[40] B. Hayes, "The phonology of rhythm in English," vol. 15, no. 1, Winter 1984, pp. 33-74.

[41] J. Schillinger, "General theory of rhythm as applied to music," Bulletin of the American Musicological Society, no. 2, June 1937, pp. 8-9.

[42] O. Wright, The Modal System of Arab and Persian Music A.D. 1250-1300, London Oriental Series - vol. 28, Oxford University Press, Oxford, 1978.

[43] O. Wright, "A preliminary version of the kitāb al-Adwār," Bulletin of the School of Oriental and African Studies, University of London, vol. 58, no. 3, 1995, pp. 455-478.

[44] G. T. Toussaint, "Elementary proofs of the hexachordal theorem," Abstracts of the Special Session on Mathematical Techniques in Music Analysis - I, 113th Annual Meeting of the American Mathematical Society, New Orleans, U.S.A., January 6, 2007.

[45] F. Benadon, "A circular plot for rhythm visualization and analysis," Music Theory Online, Vol. 13, No. 3, September 2007.

[46] I. Quinn, "Fuzzy extensions to the theory of contour," Music Theory Spectrum, vol. 19, no. 2, Autumn 1997, pp. 232-263.

[47] I. Quinn, "The combinatorial model of pitch contour," Music Perception, vol. 16, no. 4, Summer 1999, pp. 439-456.

[48] R. D. Morris, "New directions in the theory and analysis of musical contour," Music Theory Spectrum, vol. 15, no. 2, Autumn 1993, pp. 205-228.

[49] B. Colley, "A comparison of syllabic methods for improving rhythm literacy," Journal of Research in Music Education, vol. 35, no. 4, Winter 1987, pp. 221-235.

[50] D. P. Ester, J. W. Scheib, and K. J. Inks, "A rhythm system for all ages," Music Educators Journal, vol. 93, no. 2, November 2006, pp. 60-65.

[51] G. T. Toussaint, "A comparison of rhythmic similarity measures," Proceedings of ISMIR 2004: 5th International Conference on Music Information Retrieval, Universitat Pompeu Fabra, Barcelona, Spain, October 10-14, 2004, pp. 242-245.

[52] G. T. Toussaint, "Classification and phylogenetic analysis of African ternary rhythm timelines," Proceedings of BRIDGES: Mathematical Connections in Art, Music, and Science, University of Granada, Granada, Spain July 23-27, 2003, pp. 25-36.

[53] M. Senechal, "A point set puzzle revisited," European Journal of Combinatorics, vol. 29, 2008, pp. 1933-1944.

[54] J. E. Iglesias, "On Patterson's cyclotomic sets and how to count them," Zeitschrift f"ur Kristallographie, Vol. 156, 1981, pp. 187-196. 